Research Article

\title{
Tumor-Derived Exosomal miR-620 as a Diagnostic Biomarker in Non-Small-Cell Lung Cancer
}

\author{
Youyong Tang, ${ }^{1}$ Zhijun Zhang, ${ }^{2}$ Xingguo Song, ${ }^{3}$ Miao Yu, ${ }^{4}$ Limin Niu, ${ }^{1}$ Yajing Zhao, \\ Li Wang, ${ }^{1}$ Xianrang Song, ${ }^{1}$ and Li Xie $\mathbb{1}^{1}$ \\ ${ }^{1}$ Department of Clinical Laboratory, Shandong Cancer Hospital and Institute, \\ Shandong First Medical University and Shandong Academy of Medical Sciences, Jinan, China \\ ${ }^{2}$ Department of Clinical Laboratory, Taian City Central Hospital, Taian, China \\ ${ }^{3}$ Shandong Provincial Key Laboratory of Radiation Oncology, Shandong Cancer Hospital and Institute, \\ Shandong First Medical University and Shandong Academy of Medical Sciences, Jinan, China \\ ${ }^{4}$ Department of Clinical Laboratory, Shandong Provincial Third Hospital, Cheeloo College of Medicine, Shandong University, \\ Jinan, China
}

Correspondence should be addressed to Li Xie; 1_xie2001@126.com

Received 29 October 2020; Revised 16 November 2020; Accepted 20 November 2020; Published 2 December 2020

Academic Editor: Nicola Silvestris

Copyright (C) 2020 Youyong Tang et al. This is an open access article distributed under the Creative Commons Attribution License, which permits unrestricted use, distribution, and reproduction in any medium, provided the original work is properly cited.

Background. Evidence has suggested the functional role of exosomal miRNAs in cancer diagnosis. This study aimed to determine whether the serum exosomal biomarkers can improve the diagnosis of patients with non-small-cell lung cancer (NSCLC). Materials and Methods. The exosomes were extracted from the serum of NSCLC patients $(n=235)$ and healthy donors $(n=231)$ using ultracentrifugation and then were evaluated by using transmission electron microscopy, qNano, and western blotting. The serum exosomal miRNA expression was validated using qPCR. Results. Exosomal miR-620 was significantly reduced in NSCLC and early-stage NSCLC patients $(P<0.0001)$ when compared to that of healthy controls, with an area under the curve (AUC) of 0.728 and 0.707, respectively. Exosomal miR-620 expression showed an association with drinking $(P=0.008)$ and distant metastasis $(P=0.037)$. Additionally, the downregulated exosomal miR-620 showed association with chemotherapeutic effect $(P=0.044)$. Conclusion. These findings suggest the serum exosomal miR-620 as a promising diagnostic and prognostic noninvasive biomarker in NSCLC patients.

\section{Introduction}

Approximately $80 \%$ of patients suffer from non-small-cell lung cancer (NSCLC), which is the most common subtype and a major cause of morbidity and mortality worldwide [1]. The 5-year overall survival rate of individuals with local NSCLC (stage 1) was as high as $90 \%$. However, most of the NSCLC patients are diagnosed after reaching a more advanced stage, and so these patients have a relatively lower survival rate $[2,3]$. Hence, it is imperative to investigate a therapeutic approach for early screening and specific diagnosis of NSCLC patients.

Exosomes are nanoscale vesicles that have a diameter of 30-100 nm and are secreted by almost all types of cells. These were seen across many bodily fluids including urine, saliva, plasma, and malignant secretions [4]. Exosomes translate to protein, miRNA, and mRNA, playing an important role in intercellular communication [5]. Among these, the microRNAs (miRNAs) act as promising molecular markers in diagnosing the tumors [6].

MiRNAs, which are 20-24nt noncoding RNAs, function in posttranscriptional regulation of gene expression in multicellular organisms by directly binding to the $3^{\prime}$ untranslated region ( $3^{\prime} \mathrm{UTR}$ ) of their target mRNA [6]. MiRNAs have been reported to be significant predictive/ prognostic biomarkers in different cancers. Studies strongly highlight the potential role of miRNAs in pancreatic ductal adenocarcinoma tumorigenesis, diagnosis, prognosis, and 
therapy [7]. Several studies have suggested that some specific miRNAs (miR-122, miR-26, and miR-20) have a poor prognosis in patients with hepatocellular carcinoma and are related to poor response to treatment [8]. Moreover, the study showed that aberrant expression of miR-20b, miR-27a, and miR-181a was associated with chemotherapeutic response in gastric cancer suggesting a possible novel therapeutic strategy [9]. More interestingly, miRNAs packed in exosomes are shielded by RNase to prevent degradation when compared to the disposed miRNAs [10]. In addition, the miRNAs packed into exosomes pass through different bodily fluids (i.e., blood and spinal fluid) in a stable cell-free form [11]. Hence, scientists are paying much attention to explore the usefulness of miRNAs wrapped in exosomes, as these might act as possible biomarkers in diagnosis and prognosis of a disease condition [12-14]. Recently, exosomal miRNA has received much attention as a possible diagnostic biomarker in lung cancer [15]. However, to our knowledge, the relationship between exosomal miR-620 expression and the diagnosis and prognostic biomarkers of NSCLC has not yet been clarified.

Hence, in this study, miR-620 was selected for large-scale verification in the serum exosomes of NSCLC patients and healthy donors, establishing a diagnostic group that binds to cytokeratin 19 fragment (CYFRA21-1) and carcinoembryonic antigen (CEA) for the first time to achieve ideal diagnosis of NSCLC with high sensitivity and specificity.

\section{Materials and Methods}

2.1. Patients and Healthy Donors. Overall, 235 NSCLC patients and 231 healthy controls from the Shandong Cancer Hospital and Institute, Shandong First Medical University, and Shandong Academy of Medical Sciences (Jinan, China) were recruited between January 2019 and July 2019. All participants provided written informed consent forms for participating in the study. Staging of the tumor was done according to the American Cancer Council's AJCC Cancer Staging Manual (2010). Patients included in this study did not receive any anticancer therapy and had any additional immune, endocrine, or metabolic diseases. Healthy donors included had no other diseases. The characteristics of the patients are listed in Table 1.

2.2. Serum Exosomal Isolation. Ultracentrifugation was used to extract the exosomes as described in a previous study [16]. In brief, serum was centrifuged at $3000 \mathrm{~g}$ for 10 minutes, again at $10000 \mathrm{~g}$ for 30 minutes at $4^{\circ} \mathrm{C}$, and finally at 100 $000 \mathrm{~g}$ for 120 minutes at $4^{\circ} \mathrm{C}$ (Beckman Coulter) to separate $1 \mathrm{ml}$ supernatant. The pellet was then dissolved in $1 \mathrm{~mL}$ phosphate-buffered saline (PBS) or TRIzol reagent to isolate RNA.

2.3. Transmission Electron Microscopy (TEM). The exosomal beads were dripped into the grid with $50 \mu \mathrm{L}$ of $1 \%$ glutaraldehyde, placed for 5 minutes, and then transferred into $100 \mu \mathrm{L}$ of distilled water. After 2 minutes, the grid was directly transferred to $50 \mu \mathrm{L}$ uranyl-oxalate solution with a $\mathrm{pH}$
TABle 1: Characteristics of NSCLC patients for differentially expressed serum exosomal miR-620.

\begin{tabular}{|c|c|c|c|c|}
\hline Characteristics & & No. of cases & Median & $P$ value \\
\hline \multirow{2}{*}{ Age (y) } & $<62$ & 115 & 5.4500 & \multirow{2}{*}{0.235} \\
\hline & $\geq 62$ & 120 & 5.2350 & \\
\hline \multirow[b]{2}{*}{ Gender } & Male & 141 & 5.3550 & \multirow{2}{*}{0.361} \\
\hline & Female & 94 & 5.1625 & \\
\hline \multirow{2}{*}{ Smoking } & Yes & 122 & 5.3150 & \multirow{2}{*}{0.502} \\
\hline & No & 113 & 5.2800 & \\
\hline \multirow{3}{*}{ Drinking } & Yes & 76 & 5.1075 & \multirow{3}{*}{0.008} \\
\hline & No & 159 & 5.4100 & \\
\hline & $\mathrm{AC}$ & 165 & 5.3906 & \\
\hline \multirow[t]{2}{*}{ Pathology diagnosis } & SCC & 59 & 5.2500 & \multirow[t]{2}{*}{0.988} \\
\hline & Other & 11 & 5.3823 & \\
\hline \multirow{2}{*}{ Lymph node metastasis } & Yes & 106 & 5.4250 & \multirow{2}{*}{0.233} \\
\hline & No & 129 & 5.2000 & \\
\hline \multirow{2}{*}{ TNM staging } & I-II & 129 & 5.2450 & \multirow{2}{*}{0.440} \\
\hline & III-IV & 105 & 5.4100 & \\
\hline \multirow{2}{*}{ Distant metastasis } & Yes & 62 & 5.6626 & \multirow{2}{*}{0.037} \\
\hline & No & 173 & 5.2000 & \\
\hline
\end{tabular}

AC, adenocarcinoma; SCC, squamous cell carcinoma.

of 7 for 5 minutes, and then, the glass dish was covered with sealing film on the ice. The grid was cleaned using distilled water for 7 times for 2 minutes each and then evaluated by JEM-1200EX TEM (JEOL) at $100 \mathrm{kV}$.

2.4. qNano. The size and the particle concentration of exosomes were measured by using TRPS (qNano; Izon Science Ltd). The data were analyzed by using Izon Control Suite software v.3.3.2.2000 (Izon Science Ltd).

2.5. Western Blotting Analysis. The extracted protein was separated on $10 \%$ SDS-PAGE, transferred onto the PVDF membranes (Millipore), placed in blocking buffer for $2 \mathrm{~h}$, treated with primary antibody at $4^{\circ} \mathrm{C}$ overnight, and finally treated with secondary antibody at room temperature for $1 \mathrm{~h}$. In addition, the protein was detected on a film through an ECL blot detection reagent (P0018; Beyotime). The main antibodies are as follows: anti-GM130, anti-CD54, and antiCD9 (CST, America).

2.6. RNA Isolation and Real-Time Polymerase Chain Reaction $(P C R)$. The total RNA of serum exosomes was extracted using TRIzol reagent (Van Allen Way) and then converted to cDNA using the Mix-X miRNA First-Strand Synthesis Kit (Takara Bio). For qPCR, $2 \mu \mathrm{l}$ cDNA was mixed with the TBGreen Premix Ex Taq II reagent (Takara Bio) and primers in a $20 \mu \mathrm{l}$ reaction volume. qPCR analysis was done using LC480 (Roche Diagnostics, Germany). U6 served as an internal reference, and the relative expression of serum exosome miR-620 was assessed using the formula $\Delta \mathrm{CT}$ $\left(\mathrm{Ct}_{\mathrm{miRNA}^{-}} \mathrm{Ct}_{\mathrm{U} 6}\right)$ [16]. Each sample was assessed in duplicates.

2.7. Statistical Analysis. Data were analyzed using SPSS 22.0 (IBM) software and GraphPad Prism 6.0 (GraphPad software). The data were presented as mean with SD or median 
and interquartile range. The Mann-Whitney $U$ test or $t$ test was utilized for comparing the two groups. The receiver operating characteristic (ROC) curve analysis was used for discriminating the biomarkers. $P$ values of $<0.05$ were considered to be statistically significant for all comparisons.

\section{Results}

3.1. Characterization of Serum Exosomes. Serum exosomes isolated from NSCLC patients and healthy donors were characterized by TEM, qNano, and western blotting analysis. The results showed typical oval-shaped vesicles under TEM (Figure 1(a)). Correspondingly, the qNano analysis revealed that the diameters of the majority of exosomes ranged between 50 and $150 \mathrm{~nm}$ (Figure 1(b)). Additionally, the exosome protein markers, CD54, and CD9 were found to be upregulated in exosomes but not expressed in whole cell lysates. GM130 (negative control) was expressed in cell extracts and not in the extracted exosomes (Figure 1(c)).

\subsection{Characterization of Identified Serum Exosomal miR-620.}

The data of NSCLC patients for miRNA profiling have been described previously [17], in which miR-620 was selected for our next research due to its obvious change in expression. To prove the inclusion of serum miRNA in the exosomes, the expression of miR-620 in exosome-depleted supernatant (EDS) and exosomes was determined. Indeed, miR-620 expression in exosomes showed a significant increase when compared to EDS (Figure 2(a)). Moreover, the stability of exosomal miRNA indicated that miR-620 expression in exosomes showed no change after RNase A treatment (Figure 2(b)). Briefly, these results suggested that miR-620 mainly existed in the exosomes, protecting miRNAs against degradation by RNases. Besides, after leaving the exosomes at room temperature for $0,6,12,18$, and 24 hours, no significant changes were observed in the expression of miR620 (Figure 2(c)).

3.3. Exosomal miR-620 Was Decreased in NSCLC Significantly. Next, validation of exosomal miR-620 was done by RT-qPCR in independent serum samples obtained from 235 NSCLC patients and 231 healthy donors. The relationship between exosomal miR-620 expression and the clinical factors is presented in Table 1. Exosomal miR-620 expression showed association with drinking $(P=0.008$, Figure 2(d)) but not with age, gender, smoking history, and pathology $(P>0.05)$. Moreover, the relationship of exosomal miR-620 expression levels was further assessed with TNM stages. Our results revealed that the exosomal miR-620 expression levels showed significant differences in the Tstage and distant metastasis (Figures 2(e) and 2(f)) but no relation with lymph node metastasis (data not shown).

Next, differential miR-620 expression was identified between NSCLC patients and healthy donors. As depicted in Figures 2(g) and 2(h), the serum exosomal miR-620 expression was significantly downregulated in NSCLC patients
$(P<0.0001)$ as well as in early-stage NSCLC when compared with that in healthy groups. This indicated that exosomal miR-620 acts as a potentially promising NSCLC diagnostic biomarker and predictor of NSCLC metastasis.

3.4. Serum Exosomal miR-620 May Serve as NSCLCDiagnostic Marker. To evaluate the diagnostic capacity of exosomal miR-620, the AUC was calculated. Exosomal miR-620 had an AUC of 0.728 with a sensitivity of $74 \%$ and specificity of $62.3 \%$ or 0.707 with a sensitivity of $62.8 \%$ and a specificity of $68.4 \%$ when compared between healthy donors and patients with NSCLC or early NSCLC (Figures 3(a) and 3(b)), suggesting its role as a biomarker in diagnosis/early diagnosis of NSCLC.

Moreover, miR-620 with CEA (carcinoembryonic antigen) was combined to facilitate diagnostic efficiency. As shown in Figure 3(c), miR-620 has elevated the AUC of CEA significantly from 0.853 to 0.882 . Similarly, the combination of miR-620 with CYFRA21-1 led to an improvement in the diagnosis of NSCLC, as the AUC was elevated from 0.757 to 0.834 (Figure 3(d)). To obtain an improved diagnostic capacity, the exosomal miR-620 with CEA and CYFRA21-1 were merged, leading to a great improvement in the diagnostic ability of NSCLC. The AUC value was 0.900 , and the sensitivity was $73.8 \%$, while the specificity was shown to be 90.9\% (Figure 3(e)).

Consecutively, the combination of exosomal miR-620 with CEA or CYFRA21-1 showed a significant improvement in the diagnostic ability of early-stage NSCLC (AUC $=0.814$ and 0.761 , respectively) (Figures $3(\mathrm{f})$ and $3(\mathrm{~g})$ ). Next, the diagnostic ability of the combination of miR-620, CEA, and CYFRA21-1 in early-stage NSCLC patients was assessed. The AUC was 0.824 with a sensitivity of $74.4 \%$ and specificity of $74.9 \%$ (Figure 3(h)). These results enhanced our observation that exosomal miR-620 acts as a potential biomarker in diagnosis/early diagnosis of NSCLC.

3.5. Role of Exosomal miR-620 in Chemotherapeutic Response Assessment. Additionally, the relationship between serum exosomal miR-620 expression levels and efficacy of chemotherapy was evaluated. Overall, 39 NSCLC patients who received first-line chemotherapy were enrolled in this study. The ratio of patients who experienced a complete response (CR) and partial response (PR) is defined as the remission rate (RR). The proportion of patients with the best response to CR, PR, and stable disease (SD) is designated as the disease control rate (DCR) [18]. The RR of first-line chemotherapy in exosomal miR-620 was $17.9 \%$. Besides, the DCR of first-line chemotherapy in exosomal miR-620 was $79.5 \%$ (as shown in Table 2). Furthermore, these patients were separated into PR and non-PR groups. Non-PR included patients with SD and progressive disease (PD). This data suggested that exosomal miR-620 expression was reduced in the non-PR group when compared to that in the PR group $(P=0.044$, Figures $4(\mathrm{a})$ and $4(\mathrm{~b}))$, demonstrating that exosomal miR-620 might act as a 


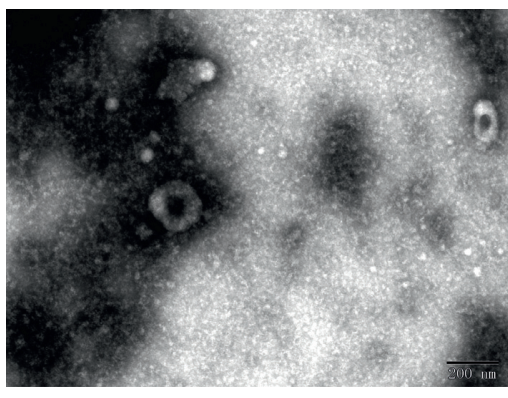

(a)

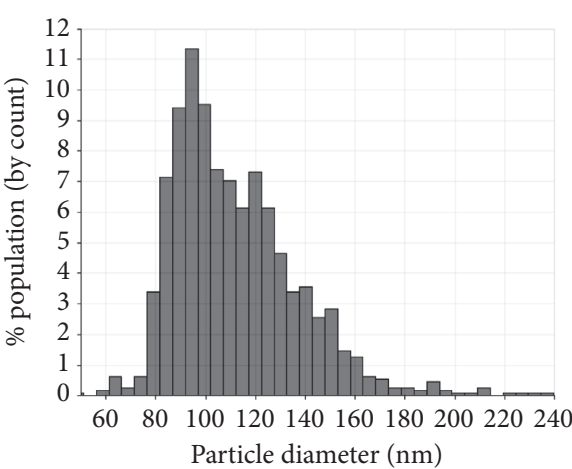

(b)

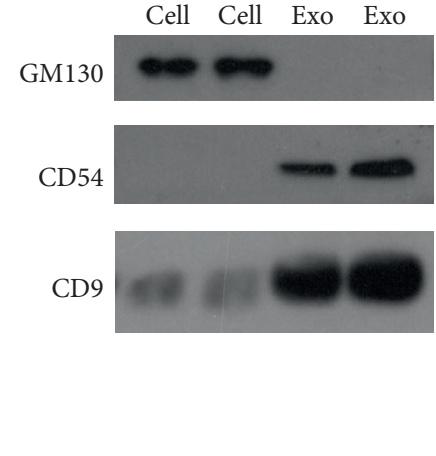

(c)

FIGURE 1: Characterization of serum exosomes. (a) TEM images indicating the characteristic data of exosomes with a diameter of 50-150 nm in NSCLC patients. (b) The exosomes with a size range of 50-150 nm were analyzed by the qNano system. (c) The exosomal protein markers, such as CD54, CD9, and GM130, were detected in the serum by western blotting analysis.

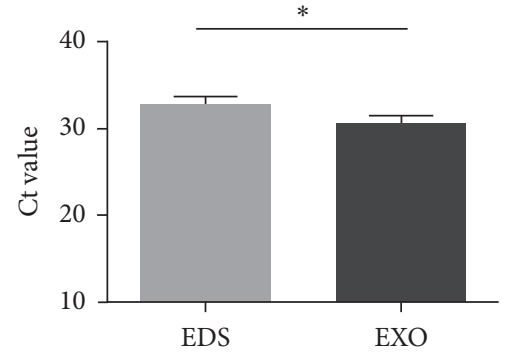

(a)

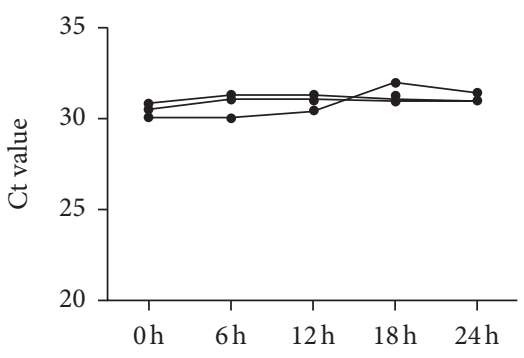

(c)

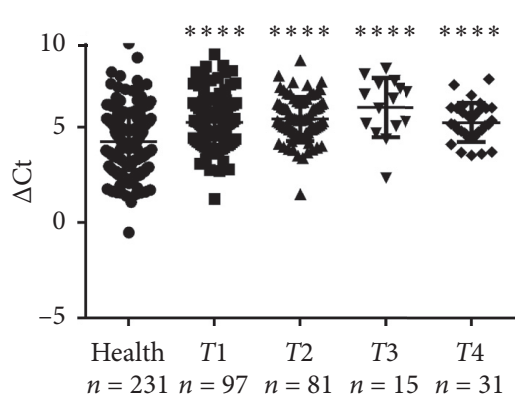

(e)

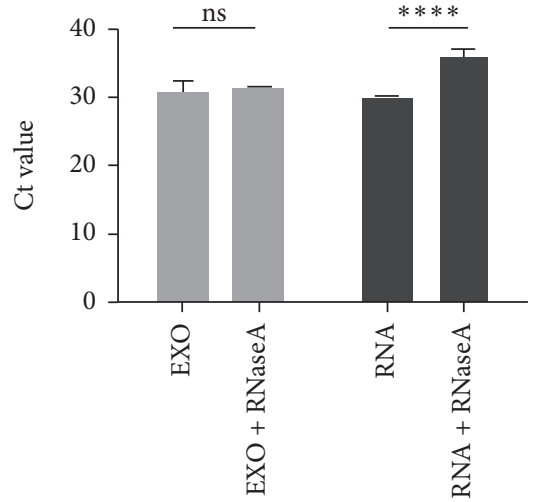

(b)

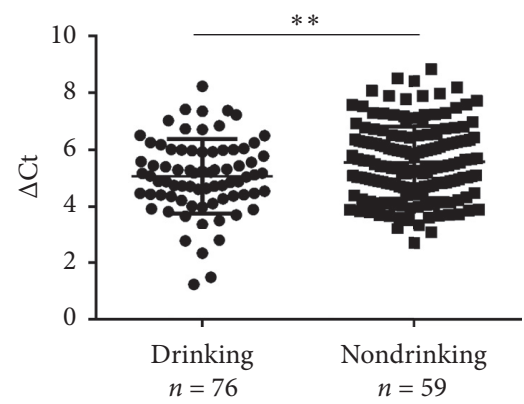

(d)

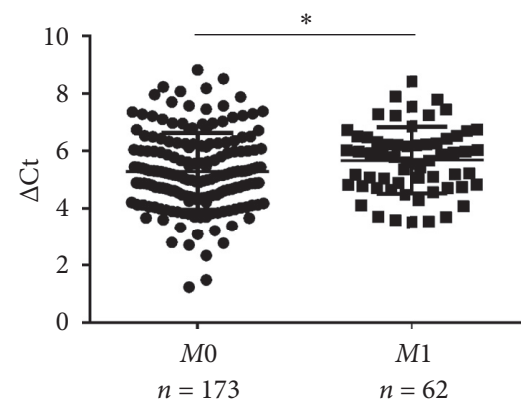

(f)

Figure 2: Continued. 


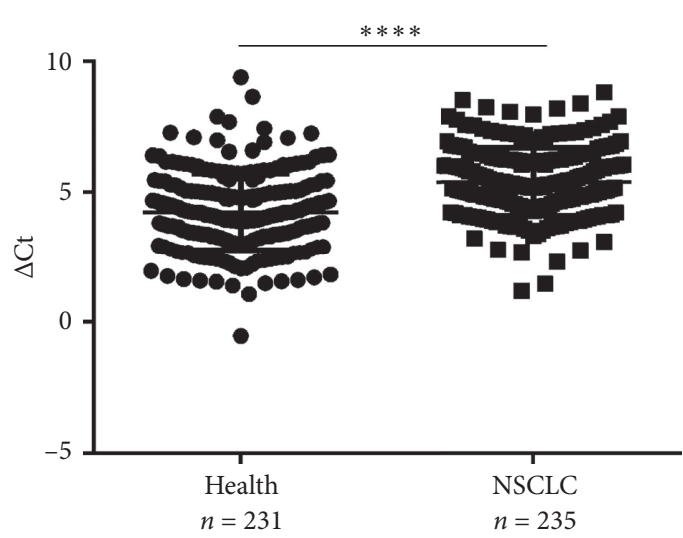

(g)

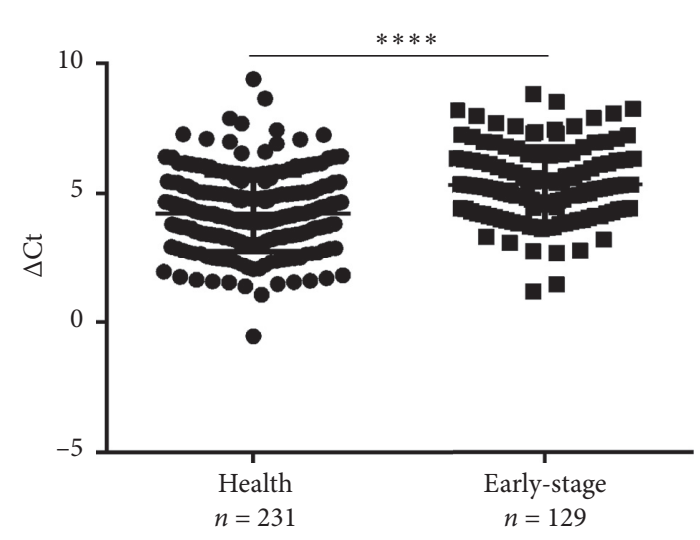

(h)

Figure 2: Exosomal miR-620 was decreased in NSCLC significantly. (a) qRT-PCR assessment of miR-620 expression levels from serum exosomes (EXO) and supernatant depleted exosomes (EDS). (b) Analysis of the expression level of miRNA-620 in RNase A-treated exosomes or serum. (c) qRT-PCR analysis of exosome miR-620 expression after incubation at room temperature. The serum exosomal miR620 demonstrated a significant relationship with (d) drinking status, (e) T stage, and (f) distant metastasis. Serum exosomal miR-620 expression in NSCLC $(G)$ or early-stage NSCLC patients $(h)$ and healthy donors were evaluated by the qRT-PCR assay $\left({ }^{*} P<0.05\right.$, ${ }^{* *} P<0.01,{ }^{* * * *} P<0.0001$, and ns: not significant).

promising biomarker in predicting the chemotherapeutic effect.

\section{Discussion}

Despite the continuous improvement of treatment strategies, NSCLC is still prone to relapse and mortality. Therefore, it is necessary to urgently identify sensitive and specific biomarkers for identifying patients with NSCLC at the early stage. In our study, the exosomal miR-620 expression levels were statistically decreased in patients with NSCLC or early NSCLC, possessing relatively high diagnostic efficiency, with an AUC of 0.728 . This indicates that the serum exosomal miR-620 has the potential to act as a biomarker in diagnosing, especially early diagnosis of NSCLC.

Several studies have identified the contribution of miRNAs in the occurrence and progression of multiple cancer types, which thus provides new strategies for tumor treatment, such as biomarkers and cancer therapeutic targets [19]. Identification of miRNAs in serum provides potential applications of these as noninvasive biomarkers for identification, monitoring, and prediction of cancer prognosis, including cancers such as colorectal cancer [20], lung cancer [21], and prostate cancer [22]. Many research studies have also indicated that miR-620 was involved in various biological processes, and aberrant expression of miR-620 showed association with varied cancer types [23, 24]. It has been reported that miR-620 is capable of inhibiting hepatocellular growth and tumorigenesis by blocking the elevation of alpha-fetoprotein [25] and participating in the biological process of colorectal cancer [26], as well as promoting tumor radio resistance through 15-hydroxyprostaglandin dehydrogenase (HPGD) [27].
In our study, several evidences were presented to verify that the exosomal miR-620 serves as a potential biomarker in NSCLC patients. Firstly, the exosomal miR-620 showed significant differences between healthy donors and patients with NSCLC or early NSCLC, indicating its role in tumorigenesis. More importantly, ROC curve analysis demonstrated that exosomal miR-620 exerted diagnostic efficiency with an AUC of 0.728 in NSCLC patients when compared to healthy individuals. Moreover, exosomal miR620 was significantly downregulated in patients with metastatic NSCLC, indicating its potential role in predicting metastasis. Finally, exosomal miR-620 expression showed an association with response to chemotherapy. Therefore, exosomal miR-620 could be utilized as a possible prognostic indicator to determine that NSCLC patients might benefit from chemotherapy.

In clinical circumstances, the use of serum-derived exosomal miRNA as a biomarker has some advantages. Firstly, the serum-derived exosomes are largely secreted from tumor cells. Compared with tumor tissues, the serum-derived exosomes could more accurately and dynamically reflect the status and function of the tumor cells. Secondly, detecting exosomal miRNAs requires only blood samples, which thereby could overcome the spatial diversity of tissue samples and help to monitor the progression of tumors in a timely manner throughout the treatment process. Furthermore, due to the double membrane structure, miRNAs in exosomes are more stable than those in blood.

However, there are many limitations in this study that should be considered carefully. Firstly, the sample size of NSCLC patients is relatively small. In the future, a larger study with more patients might confirm these findings. 


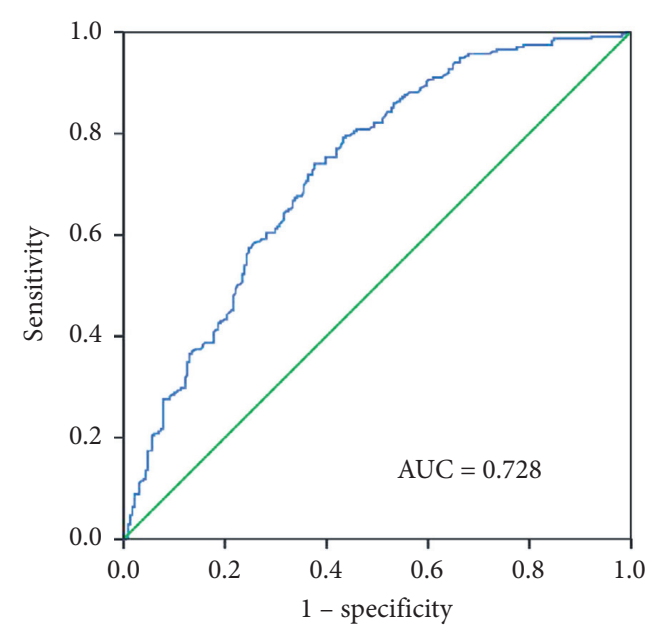

(a)

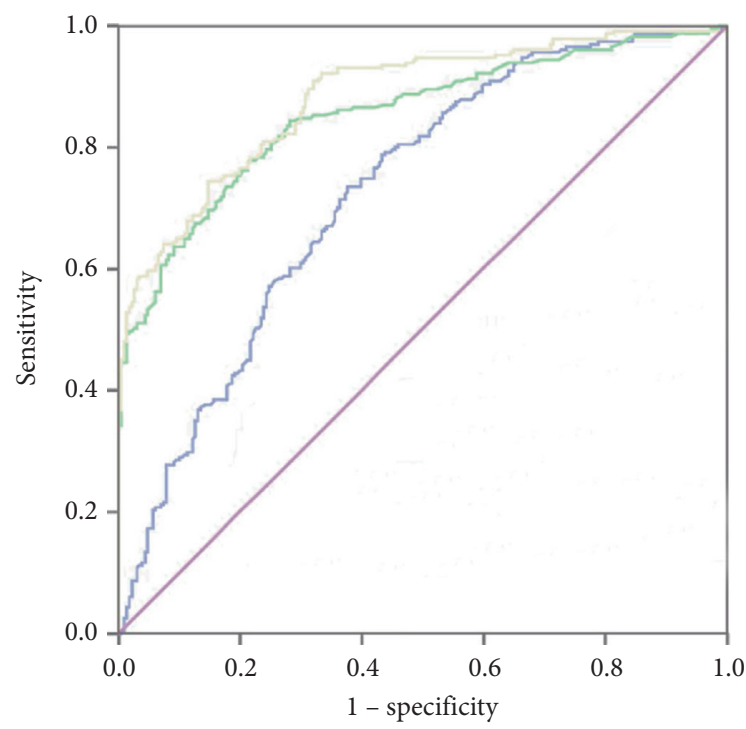

Source of the curve

- $\mathrm{AUC}=0.882$

- miR-620

_ Predicted probability

_ Reference line

(c)

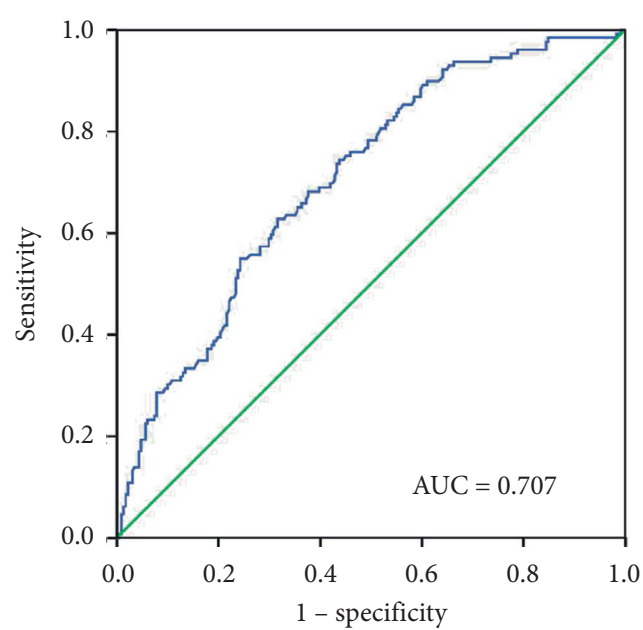

(b)

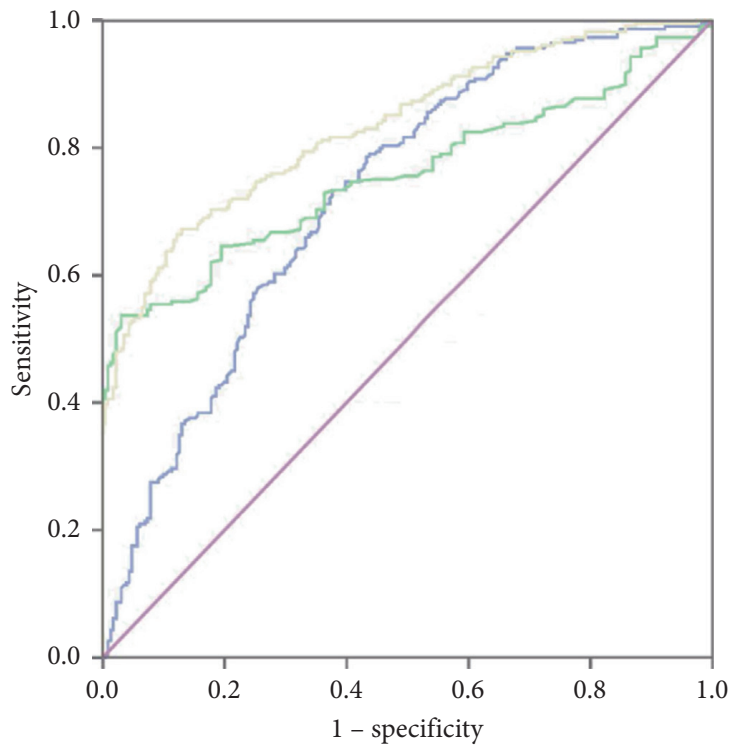

Source of the curve

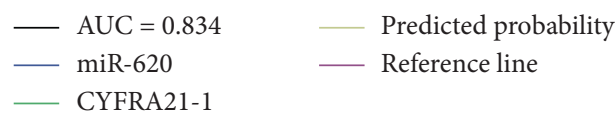

(d)

FIgURE 3: Continued. 


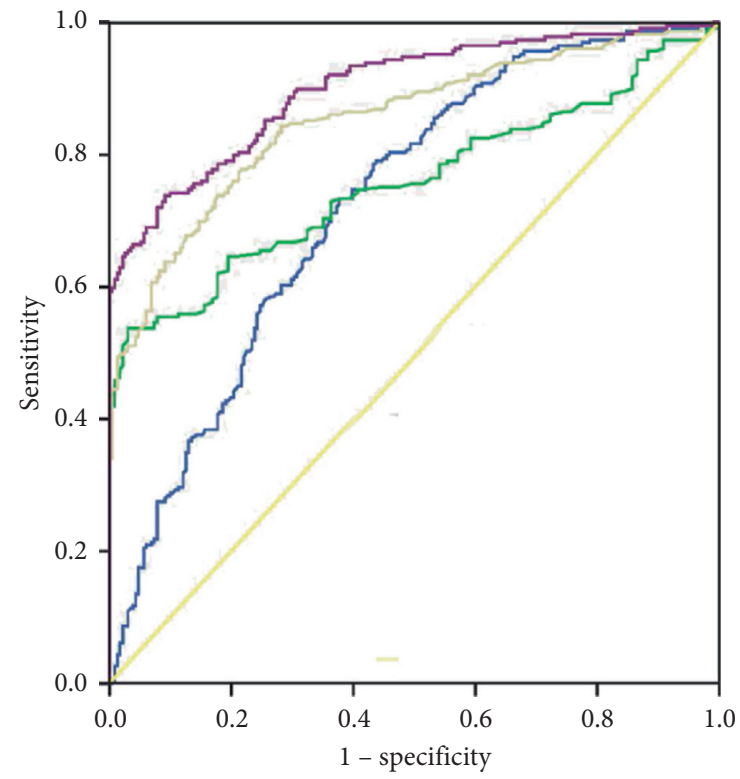

Source of the curve

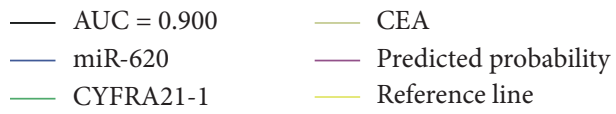

(e)

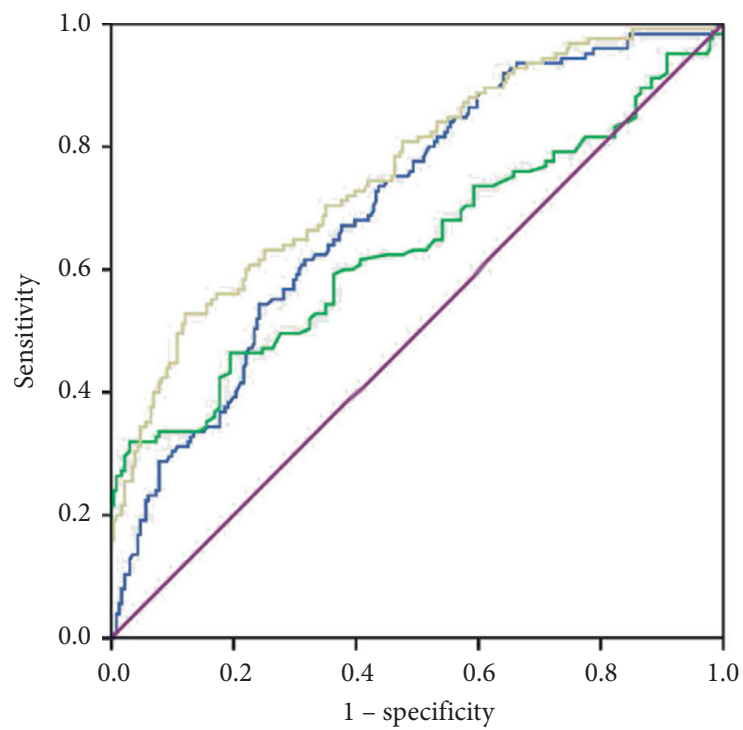

Source of the curve

$\begin{array}{ll}\text { AUC }=0.761 & \text { Predicted probability } \\ \text { miR-620 } & \text { Reference line } \\ \text { CYFRA21-1 } & \end{array}$

(g)

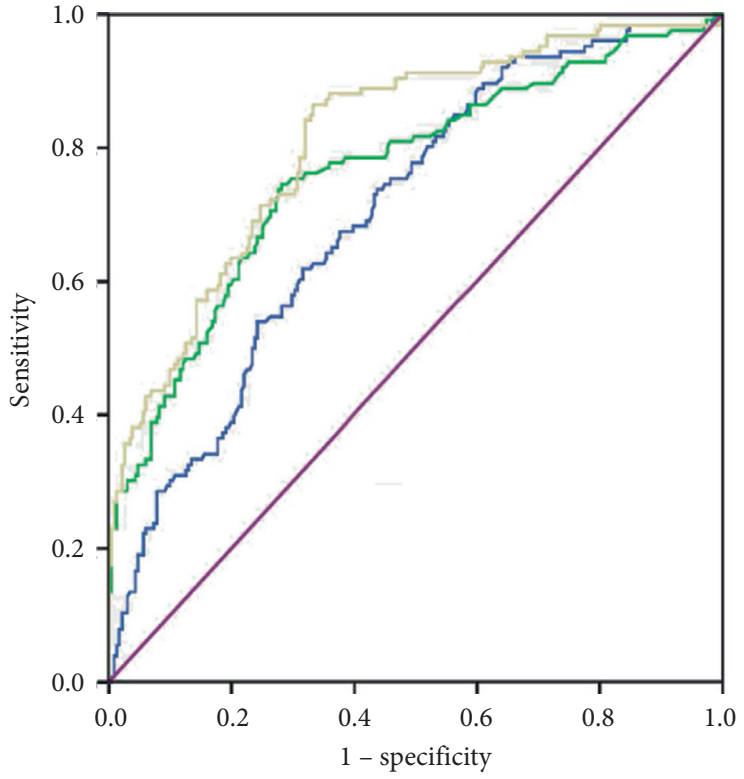

Source of the curve

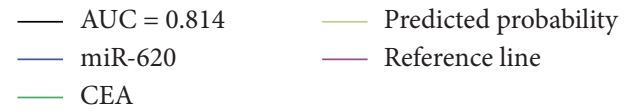

(f)

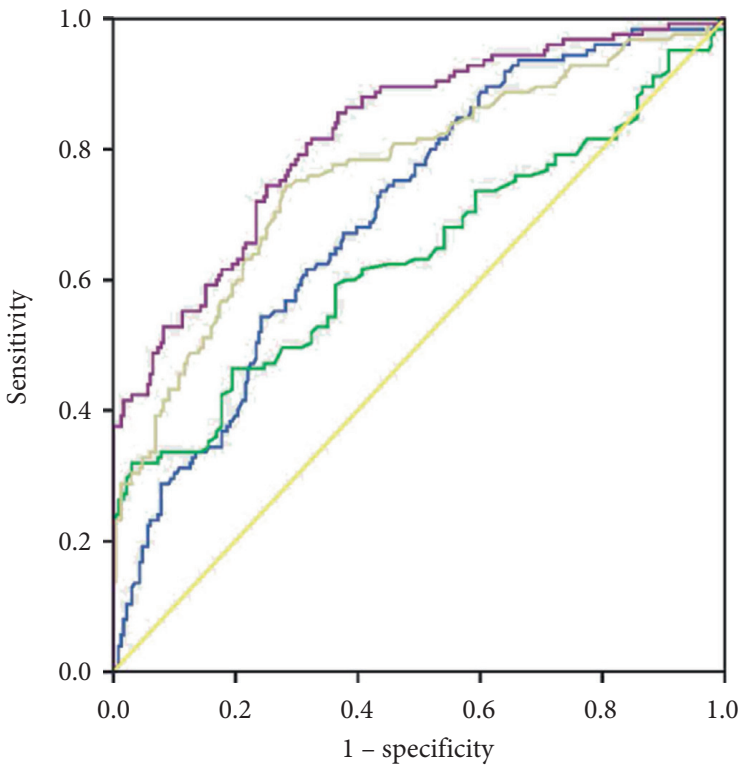

Source of the curve

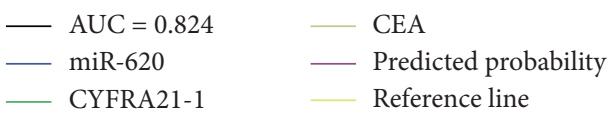

(h)

Figure 3: Exosomal miR-620 acts as a diagnostic marker in NSCLC patients. The AUC of serum exosomal miR-620 in patients with NSCLC or early-stage NSCLC and healthy donors was 0.728 (a) or 0.707 (b), respectively. The AUC of serum exosome miR-620 combined with CEA or CYFRA21-1 in NSCLC patients and healthy donors was 0.882 (c) or 0.834 (d), respectively. (e) The AUC of serum exosomal miR-620 in combination with CEA and CYFRA21-1 was 0.900 in NSCLC patients and healthy donors. The AUC of serum exosome miR-620 in combination with CEA or CYFRA21-1 in early NSCLC patients and healthy donors was 0.814 (f) or 0.761 (g), respectively. (h) The AUC of serum exosomal miR-620 in combination with CEA and CYFRA21-1 was 0.824 in early-stage NSCLC patients as well as healthy donors. 
TABLe 2: Response to the first chemotherapy in serum exosomal miR-620.

\begin{tabular}{lccccccc}
\hline Gene & $\mathrm{N}$ & CR & PR & SD & PD & RR\% & DCR\% \\
\hline miR-620 & 39 & 0 & 7 & 24 & 8 & 17.9 & 79.5 \\
\hline
\end{tabular}

CR: complete response, PR: partial response, SD; stable disease, PD: progressive disease, RR: response rate; DCR: disease control rate.

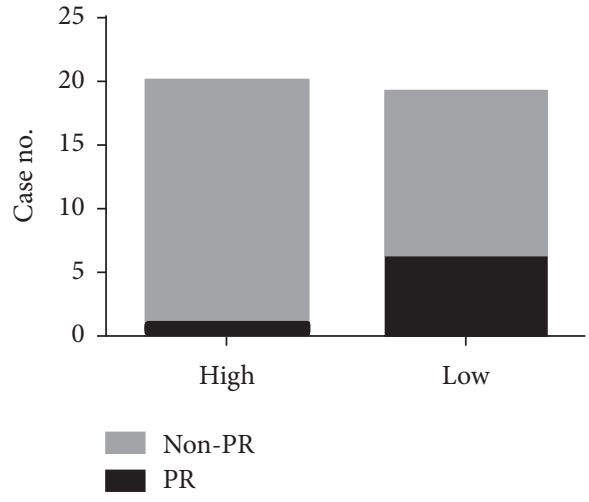

(a)

\begin{tabular}{cccccc}
\hline & & PR & Non-PR & Total & $P$ value \\
\hline \multirow{3}{*}{ miR-620 } & High & 1 & 19 & 20 & \\
& Low & 6 & 13 & 19 & 0.044 \\
& Total & 7 & 32 & 39 & \\
\hline
\end{tabular}

(b)

FIGURE 4: Role of exosomal miR-620 in chemotherapeutic effect prediction.

Secondly, the specific mechanism and specific function of exosomal miR-620 in different stages of NSCLC have not been evaluated.

In conclusion, this study demonstrated that serum exosomal miRNAs might have diagnostic and prognostic values for diagnosing NSCLC, thus providing novel opportunities as a noninvasive technique and treatment strategy.

\section{Data Availability}

The datasets used and analyzed during the current study are available from the corresponding author on reasonable request.

\section{Ethical Approval}

The studies involving human participants were reviewed and approved by the Shandong Cancer Hospital Affiliated to Shandong First Medical University and Shandong Academy of Medical Sciences of committee (SDTHEC201703017).

\section{Consent}

All subjects gave written informed consent in accordance with the Declaration of Helsinki.

\section{Conflicts of Interest}

The authors declare that there are no conflicts of interest.

\section{Acknowledgments}

This work was supported by the National Natural Science Foundation of China (81972014, 81672104, and 8150111724), the Shandong Provincial Key Research and
Development Program (2017CXGC1207 and 2016GSF201146), and Taishan Scholars Program of Shandong Province (ts20120505).

\section{References}

[1] R. L. Siegel, K. D. Miller, and A. Jemal, "Cancer statistics, 2020," CA: A Cancer Journal for Clinicians, vol. 70, no. 1, pp. 7-30, 2020.

[2] C. Zappa and S. A. Mousa, "Non-small cell lung cancer: current treatment and future advances," Translational Lung Cancer Research, vol. 5, no. 3, pp. 288-300, 2016.

[3] J. C. Simeone, B. L. Nordstrom, K. Patel, and A. B. Klein, "Treatment patterns and overall survival in metastatic nonsmall-cell lung cancer in a real-world, US setting," Future Oncology, vol. 15, no. 30, pp. 3491-3502, 2019.

[4] C. Lässer, V. Seyed Alikhani, K. Ekström et al., "Human saliva, plasma and breast milk exosomes contain RNA: uptake by macrophages," Journal of Translational Medicine, vol. 9, no. 1, p. 9, 2011.

[5] J. Li, K. Liu, Y. Liu et al., "Exosomes mediate the cell-to-cell transmission of IFN- $\alpha$-induced antiviral activity," Nature Immunology, vol. 14, no. 8, pp. 793-803, 2013.

[6] M. Tsukamoto, H. Iinuma, T. Yagi, K. Matsuda, and Y. Hashiguchi, "Circulating exosomal MicroRNA-21 as a biomarker in each tumor stage of colorectal cancer," Oncology, vol. 92, no. 6, pp. 360-370, 2017.

[7] O. Brunetti, A. Russo, A. Scarpa et al., "MicroRNA in pancreatic adenocarcinoma: predictive/prognostic biomarkers or therapeutic targets?" Oncotarget, vol. 6, no. 27, pp. 23323-23341, 2015.

[8] A. Gnoni, D. Santini, M. Scartozzi et al., "Hepatocellular carcinoma treatment over sorafenib: epigenetics, microRNAs and microenvironment. Is there a light at the end of the tunnel?" Expert Opinion on Therapeutic Targets, vol. 19, no. 12, pp. 1623-1635, 2015. 
[9] K. Danza, N. Silvestris, G. Simone et al., "Role of miR-27a, miR-181a and miR-20b in gastric cancer hypoxia-induced chemoresistance," Cancer Biology and Therapy, vol. 17, no. 4, pp. 400-406, 2016.

[10] Y. Koga, M. Yasunaga, Y Moriya et al., "Exosome can prevent RNase from degrading microRNA in feces," Journal of Gastrointestinal Oncology, vol. 2, no. 4, pp. 215-222, 2011.

[11] Q. Fan, L. Yang, X. Zhang et al., "The emerging role of exosome-derived non-coding RNAs in cancer biology," Cancer Letters, vol. 414, pp. 107-115, 2018.

[12] T. Matsumura, K. Sugimachi, H. Iinuma et al., "Exosomal microRNA in serum is a novel biomarker of recurrence in human colorectal cancer," British Journal of Cancer, vol. 113, no. 2, pp. 275-281, 2015.

[13] D. P. Joyce, M. J. Kerin, and R. M. Dwyer, "Exosome-encapsulated microRNAs as circulating biomarkers for breast cancer," International Journal of Cancer, vol. 139, no. 7, pp. 1443-1448, 2016.

[14] Z. Zhao, Y. Yang, Y. Zeng, and M. He, "A microfluidic ExoSearch chip for multiplexed exosome detection towards blood-based ovarian cancer diagnosis," Lab on a Chip, vol. 16, no. 3, pp. 489-496, 2016.

[15] X. Jin, Y. Chen, H. Chen et al., "Evaluation of tumor-derived exosomal miRNA as potential diagnostic biomarkers for early-stage non-small cell lung cancer using next-generation sequencing," Clinical Cancer Research, vol. 23, no. 17, pp. 5311-5319, 2017.

[16] Y. Tang, Y. Zhao, X. Song, X. Song, L. Niu, and L. Xie, "Tumor-derived exosomal miRNA-320d as a biomarker for metastatic colorectal cancer," Journal of Clinical Laboratory Analysis, vol. 33, no. 9, Article ID e23004, 2019.

[17] N. Wang, W. Guo, X. Song et al., "Tumor-associated exosomal miRNA biomarkers to differentiate metastatic vs. nonmetastatic non-small cell lung cancer," Clinical Chemistry and Laboratory Medicine, vol. 58, no. 9, pp. 1535-1545, 2020.

[18] R. S. Finn, C. Bengala, N. Ibrahim et al., "Dasatinib as a single agent in triple-negative breast cancer: results of an open-label phase 2 study," Clinical Cancer Research, vol. 17, no. 21, pp. 6905-6913, 2011.

[19] I. Berindan-Neagoe, P. D. C. Monroig, B. Pasculli, and G. A. Calin, "MicroRNAome genome: a treasure for cancer diagnosis and therapy," CA: A Cancer Journal for Clinicians, vol. 64, no. 5, pp. 311-336, 2014.

[20] M. Hollis, K. Nair, A. Vyas, L. S. Chaturvedi, S. Gambhir, and D. Vyas, "MicroRNAs potential utility in colon cancer: early detection, prognosis, and chemosensitivity," World Journal of Gastroenterology, vol. 21, no. 27, pp. 8284-8292, 2015.

[21] A. Matikas, K. N. Syrigos, and S. Agelaki, "Circulating biomarkers in non-small-cell lung cancer: current status and future challenges," Clinical Lung Cancer, vol. 17, no. 6, pp. 507-516, 2016.

[22] P. S. Mitchell, R. K. Parkin, E. M. Kroh et al., "Circulating microRNAs as stable blood-based markers for cancer detection," Proceedings of the National Academy of Sciences, vol. 105, no. 30, pp. 10513-10518, 2008.

[23] Z. Zhao, C. Han, J. Liu, C. Wang, Y. Wang, and L. Cheng, "GPC5, a tumor suppressor, is regulated by miR-620 in lung adenocarcinoma," Molecular Medicine Reports, vol. 9, no. 6, pp. 2540-2546, 2014.

[24] X. Li and M. Shen, "Circular RNA hsa_circ_103809 suppresses hepatocellular carcinoma proliferation and invasion by sponging miR-620," European Review for Medical and Pharmacological Sciences, vol. 23, no. 2, pp. 555-566, 2019.
[25] C. Zhang, H. Li, W. Jiang, X. Zhang, and G. Li, "Icaritin inhibits the expression of alpha-fetoprotein in hepatitis B virus-infected hepatoma cell lines through post-transcriptional regulation," Oncotarget, vol. 7, no. 50, pp. 83755-83766, 2016.

[26] N. El-Murr, Z. Abidi, K. Wanherdrick et al., "MiRNA genes constitute new targets for microsatellite instability in colorectal cancer," PLoS One, vol. 7, no. 2, Article ID e31862, 2012.

[27] X. Huang, S. Taeb, S. Jahangiri et al., "miR-620 promotes tumor radioresistance by targeting 15-hydroxyprostaglandin dehydrogenase (HPGD)," Oncotarget, vol. 6, no. 26, pp. 22439-22451, 2015. 